\title{
Guaranteed nonlinear state estimator for cooperative systems
}

\author{
Michel Kieffer and Eric Walter \\ LSS - CNRS - Supélec - Université Paris-Sud \\ Plateau de Moulon, F-91192 Gif-sur-Yvette, France
}

\begin{abstract}
This paper is about state estimation for continuous-time nonlinear models, in a context where all uncertain variables can be bounded. More precisely, cooperative models are considered, i.e., models that satisfy some constraints on the signs of the entries of the Jacobian of their dynamic equation. In this context, interval observers and a guaranteed recursive state estimation algorithm are combined to enclose the state at any given instant of time in a subpaving. The approach is illustrated on the state estimation of a waste-water treatment process.
\end{abstract}

Keywords: bounded errors, cooperative models, interval observer, nonlinear models, set estimator, state estimator

\section{Introduction}

This paper is about state estimation for continuous-time nonlinear models, in a context where all uncertain variables can be bounded. The system under study is described by

$$
\mathbf{x}^{\prime}=\frac{d \mathbf{x}}{d t}=\mathbf{f}(\mathbf{x}, \mathbf{p}, \mathbf{w}, \mathbf{u}), \mathbf{x}(0)=\mathbf{x}_{0},
$$

where $\mathbf{x}$ is the state vector, $\mathbf{p}$ is a vector of uncertain parameters, $\mathbf{w}$ is a bounded vector of state perturbations and $\mathbf{u}$ is the known input vector. Experimental data are collected at discrete measurement times $t_{i}, i=1, \ldots, N$, according to

$$
\mathbf{y}\left(t_{i}\right)=\mathbf{h}\left(\mathbf{x}\left(t_{i}\right)\right)+\mathbf{v}\left(t_{i}\right),
$$

where $\mathbf{y}\left(t_{i}\right)$ is the measured output vector and $\mathbf{v}\left(t_{i}\right)$ the bounded measurement noise at $t_{i}$. The functions $\mathbf{f}$ and $\mathbf{h}$ may be nonlinear, so the class of models that can be considered is fairly large. The model, however, must satisfy constraints on the signs of the entries of $\partial \mathbf{f} / \partial \mathbf{x}$. These constraints are very frequently satisfied by the models used for instance in pharmacokinetics, biology, chemistry and population dynamics.

When $\mathbf{f}$ and $\mathbf{h}$ are linear functions of the state vector, many tools are available in the context of bounded errors, see, e.g., [2], [17] and [20]. In a nonlinear context, the methodology is far less developed. When uncertainty is explicitly taken into account, this is most often by resorting to linearization and using an extended Kalman filter [3], based on the linearization of (1) around the state trajectory. It is well known that this type of filter may fail to produce a useful estimate of the state vector, and that the characterization of the uncertainty in this estimate is not reliable.

An attractive alternative is guaranteed state bounding, considered in a nonlinear discretetime context in [10] and [16]. All state vectors consistent with the data, the model equations and bounds on uncertain quantities are enclosed in a subpaving, consisting of a union of disconnected boxes. In a continuous-time context, a state estimator for models such as that described by (1) - (2) has been proposed in [9] but with no state perturbation taken into account. Sets 
are again described using subpavings, and guaranteed numerical integration is performed using techniques developped to solve initial value problems, such as those described in [21]. Techniques bounding the state of systems with poorly known state equations and inputs are presented in [1] and [4], with applications in waste processing. These results require the same assumptions about the signs of the entries of $\partial \mathbf{f} / \partial \mathbf{x}$ as in this paper, which make it possible to build interval observers. An interval observer is a pair of classical observers computing a box enclosure of the state $\mathbf{x}$, provided that lower and upper bounds are available for each of the uncertain variables.

The main idea of this paper is to combine interval observers and the recursive state estimation algorithm presented in [11] and [16] to enclose the state $\mathbf{x}(t)$ of the model (1) - (2) at any given instant of time $t$ in a subpaving. The result may thus be much more accurate than in [1] and [4] where a single box enclosing all possible state vectors is computed. Computations are performed recursively and can be implemented in real time.

Recursive causal bounded-error state estimation is presented in Section 2. An idealized algorithm is first proposed in Section 2.1. The link between interval observers, cooperative systems and set observers is then presented in Sections 2.2 and 2.3, before describing an implementable counterpart to the idealized algorithm in Section 2.4. The advantages and limitations of the approach are illustrated on an example in Section 3.

\section{Recursive causal bounded-error state estimation}

Consider the model $(1)-(2)$ and a set of sampling instants $\mathcal{T}=\left\{t_{i}\right\}_{i \in \mathbb{N}}$, such that $t_{i+1}>t_{i}$, at which the measurements $\mathbf{y}\left(t_{i}\right)$ have been collected. At $t_{0}, \mathbf{x}\left(t_{0}\right)$ is only known to belong to some box $\left[\mathbf{x}_{0}\right]$. The vector $\mathbf{p}$ of uncertain parameters is assumed to be constant and to belong to some known box $[\mathbf{p}]$. The state perturbation $\mathbf{w}(t)$ is assumed to satisfy $\underline{\mathbf{w}}(t) \leqslant \mathbf{w}(t) \leqslant \overline{\mathbf{w}}(t)$ at any $t \geqslant t_{0}$, where $[\mathbf{w}(t)]=[\underline{\mathbf{w}}(t), \overline{\mathbf{w}}(t)]$ is known for all $t$ and the inequalities are to be understood componentwise. Moreover, the measurement noise $\mathbf{v}\left(t_{i}\right)$ is assumed to belong to $\left[\mathbf{v}\left(t_{i}\right)\right]=\left[\underline{\mathbf{v}}\left(t_{i}\right), \overline{\mathbf{v}}\left(t_{i}\right)\right]$, known at each $t_{i}$. Let $\mathcal{I}(t)$ be the information available at time $t \geqslant t_{0}$

$$
\mathcal{I}(t)=\left\{\left[\mathbf{x}_{0}\right],[\mathbf{p}],\{[\mathbf{w}(\tau)], \mathbf{u}(\tau)\}_{\tau \in\left[t_{0}, t\right]},\left\{\left[\mathbf{v}\left(t_{i}\right)\right]\right\}_{i=0}^{N}\right\}
$$

where $t_{N}$ is such that $t_{N} \leqslant t<t_{N+1}$. In this context, causal state estimation is the caracterization of the set $\mathcal{X}(t)$ of all values of the state $\mathbf{x}(t)$ at any time $t \geqslant t_{0}$ that are consistent with $\mathcal{I}(t)$.

\subsection{Idealized algorithm}

As the Kalman filter, the idealized recursive causal state estimator consists of two steps.

For the prediction step, assume that $\mathcal{X}\left(t_{i}\right) \subset \mathcal{D}$ is some set guaranteed to contain $\mathbf{x}\left(t_{i}\right)$. For a given $\mathbf{x} \in \mathcal{X}\left(t_{i}\right)$, let $\phi(\mathbf{x}, t, \mathbf{p}, \mathbf{w}(t), \mathbf{u}(t))$ be the flow associated with (1). Define the predicted set $\mathcal{X}^{+}\left(t_{i+1}\right)$ as

$$
\mathcal{X}^{+}\left(t_{i+1}\right)=\left\{\phi\left(\mathbf{x}, t_{i+1}, \mathbf{p}, \mathbf{w}(t), \mathbf{u}(t)\right) \mid \mathbf{p} \in[\mathbf{p}], \mathbf{w}(t) \in[\mathbf{w}(t)], \mathbf{x} \in \mathcal{X}\left(t_{i}\right)\right\} .
$$

By construction, $\mathbf{x}\left(t_{i+1}\right) \in \mathcal{X}^{+}\left(t_{i+1}\right)$.

Now, for the correction step, let $\mathcal{Y}\left(t_{i+1}\right)$ be the set of all possible values of the noise-free output, when the value of the measured output is $\mathbf{y}\left(t_{i+1}\right)$

$$
\mathcal{Y}\left(t_{i+1}\right)=\mathbf{y}\left(t_{i+1}\right)-\left[\mathbf{v}\left(t_{i+1}\right)\right]=\left\{\mathbf{y}\left(t_{i+1}\right)-\mathbf{v} \mid \mathbf{v} \in[\mathbf{v}]\left(t_{i+1}\right)\right\},
$$

and let $\mathcal{X}^{O}\left(t_{i+1}\right)$ be the set of all values of the state at time $t_{i+1}$ that could have led to an observation $\mathbf{y}$ in $\mathcal{Y}\left(t_{i+1}\right)$

$$
\mathcal{X}^{O}\left(t_{i+1}\right)=\mathbf{h}^{-1}\left(\mathcal{Y}\left(t_{i+1}\right)\right)=\left\{\mathbf{x} \in \mathbb{R}^{n} \mid \mathbf{h}(\mathbf{x}) \in \mathcal{Y}\left(t_{i+1}\right)\right\} .
$$

Then, the corrected set

$$
\mathcal{X}\left(t_{i+1}\right)=\mathcal{X}^{+}\left(t_{i+1}\right) \cap \mathcal{X}^{O}\left(t_{i+1}\right)
$$




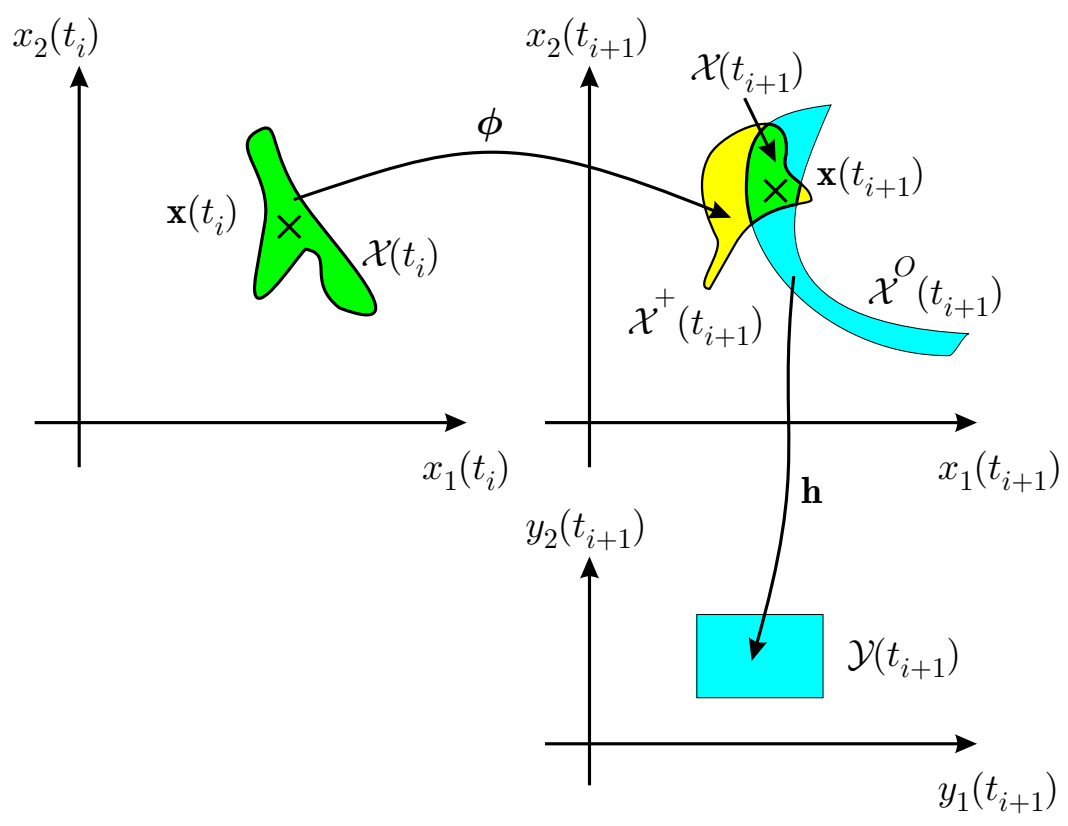

Figure 1: Idealized state estimation algorithm

is also guaranteed to contain $\mathbf{x}\left(t_{i+1}\right)$ (see Figure 1 ).

This is summarized in the following idealized algorithm.

\section{Algorithm 1}

For $i=0$ to $N$, do \{

1. Prediction: evaluate $\mathcal{X}^{+}\left(t_{i+1}\right)$;

2. Correction: $\left.\mathcal{X}\left(t_{i+1}\right)=\mathcal{X}^{+}\left(t_{i+1}\right) \cap \mathcal{X}^{O}\left(t_{i+1}\right) ;\right\}$

It is easy to show [14] that $\mathcal{X}(t)$ as evaluated by Algorithm 1 is the smallest set guaranteed to contain $\mathbf{x}(t)$ that can be computed from $\mathcal{I}(t)$ and $(1)$.

To obtain an implementable counterpart to Algorithm 1, three main problems have to be solved. The first one is to represent the sets $\mathcal{X}\left(t_{i}\right), \mathcal{X}^{O}\left(t_{i}\right)$ and $\mathcal{X}^{+}\left(t_{i}\right)$ in computer memory. The characterization of sets using subpavings described in [15] is used. The second problem is the evaluation of the inverse image of a set by a given function during the correction step (6). An outer approximation $\widehat{\mathcal{X}}^{O}\left(t_{i+1}\right)$ of $\mathcal{X}^{O}\left(t_{i+1}\right)$ by a subpaving is obtained using the SIVIA algorithm (see [12] and [13]). The precision of this outer approximation is controlled by a precision factor $\varepsilon_{S}$.

The remaining problem is the solution at the prediction step of the set of IVPs required to evaluate $\mathcal{X}^{+}\left(t_{i+1}\right)$. Standard guaranteed tools are available to solve IVP such as

$$
\left\{\mathbf{x}^{\prime}=\mathbf{f}(\mathbf{x}, t), \mathbf{x}(0)=\mathbf{x}\right\}
$$

or

$$
\left\{\mathbf{x}^{\prime}=\mathbf{f}(\mathbf{x}, t), \mathbf{x}(0) \in[\mathbf{x}]\right\},
$$

see, e.g., AWA ([18], [19]), COSY ([7], [8]) or VNODE ([21]). These techniques are based on the Picard theorem and build a Taylor expansion of the solution while bounding the remainder in a guaranteed way. These techniques, however, become very inefficient in the presence of unknown parameters or bounded state perturbations because the bounds on the remainder soon become extremely large. The next section will present a more efficient approach, based on cooperativity. 


\subsection{Interval observers and cooperative systems}

An interval observer, as defined in [4], is a pair of dynamical systems

$$
\left\{\begin{array}{l}
\underline{\mathbf{x}}^{\prime}=\underline{\mathbf{f}}(\underline{\mathbf{x}}, t) \\
\overline{\mathbf{x}}^{\prime}=\mathbf{\overline { \mathbf { f } }}(\overline{\mathbf{x}}, t)
\end{array}\right.
$$

such that $\underline{\mathbf{x}}(t) \leqslant \mathbf{x}(t) \leqslant \overline{\mathbf{x}}(t)$, for all $t \geqslant 0$, where $\mathbf{x}(t)$ is the solution of (1). At any $t \geqslant 0$, an interval observer thus provides a box $[\mathbf{x}(t)]$ enclosing the actual value of the state $\mathbf{x}(t)$. The main difficulty is to obtain an interval observer that gives tight enclosures, i.e., such that the width of the box $[\mathbf{x}(t)]$ is as small as possible. Such tight enclosures are easily obtained for monotone dynamical systems.

Definition 1 The dynamical system

$$
\mathbf{x}^{\prime}=\mathbf{f}(\mathbf{x}, t)
$$

with initial condition $\mathbf{x}(0)=\mathbf{x}_{0}$ and associated flow $\mathbf{x}(t)=\phi\left(\mathbf{x}_{0}, t\right)$ is monotone if $\mathbf{x}_{0} \leqslant \mathbf{y}_{0} \Longrightarrow$ $\phi\left(\mathbf{x}_{0}, t\right) \leqslant \phi\left(\mathbf{y}_{0}, t\right)$, for all $t \geqslant 0$.

Cooperative systems form a class of monotone dynamical systems [22].

Definition 2 The dynamical system (9), where $\mathbf{f}(\mathbf{x}, t)$ is supposed to be continuous and differentiable, is cooperative on a domain $\mathcal{D}$ if

$$
\frac{\partial f_{i}}{\partial x_{j}} \geqslant 0, \text { for all } i \neq j, t \geqslant 0 \text { and } \mathbf{x} \in \mathcal{D} \text {. }
$$

If a dynamical system such as (9), with initial condition $\mathbf{x}(0)$ only known to belong to the box $\left[\underline{\mathbf{x}}_{0}, \overline{\mathbf{x}}_{0}\right]$, is cooperative, then an interval observer may be defined by

$$
\left\{\begin{array}{l}
\underline{\mathbf{x}}^{\prime}=\mathbf{f}(\underline{\mathbf{x}}, t) \\
\overline{\mathbf{x}}^{\prime}=\mathbf{f}(\overline{\mathbf{x}}, t)
\end{array}\right.
$$

with $\underline{\mathbf{x}}(0)=\underline{\mathbf{x}}_{0}$ and $\overline{\mathbf{x}}(0)=\overline{\mathbf{x}}_{0}$. Interval observers can also be built for more complex systems, such as that described by (1).

Theorem 1 (see [22]) If there exists a pair of cooperative systems

$$
\left\{\begin{array}{l}
\underline{\mathbf{x}}^{\prime}=\underline{\mathbf{f}}(\underline{\mathbf{x}}, t), \\
\overline{\mathbf{x}}^{\prime}=\overline{\mathbf{f}}(\overline{\mathbf{x}}, t),
\end{array}\right.
$$

satisfying

- $\underline{\mathbf{f}}(\mathbf{x}, t) \leqslant \mathbf{f}(\mathbf{x}, \mathbf{p}, \mathbf{w}, \mathbf{u}) \leqslant \overline{\mathbf{f}}(\mathbf{x}, t)$, for any $\mathbf{p} \in[\underline{\mathbf{p}}, \overline{\mathbf{p}}], \mathbf{w}(t) \in[\underline{\mathbf{w}}(t), \overline{\mathbf{w}}(t)], t \geqslant 0$ and $\mathbf{x} \in \mathcal{D}$,

- $\underline{\mathbf{x}}_{0} \leqslant \mathbf{x}(0) \leqslant \overline{\mathbf{x}}_{0}$,

then the state of the system (1) satisfies

$$
\underline{\mathbf{x}}(t) \leqslant \mathbf{x}(t) \leqslant \overline{\mathbf{x}}(t), \text { for any } t \geqslant 0
$$

where $\underline{\mathbf{x}}(t)=\underline{\phi}\left(\underline{\mathbf{x}}_{0}, t\right)$ is the flow associated with $\left\{\underline{\mathbf{x}}^{\prime}=\underline{\mathbf{f}}(\underline{\mathbf{x}}, t), \underline{\mathbf{x}}(0)=\underline{\mathbf{x}}_{0}\right\}$ and $\overline{\mathbf{x}}(t)=\bar{\phi}\left(\overline{\mathbf{x}}_{0}, t\right)$ is the flow associated with $\left\{\overline{\mathbf{x}}^{\prime}=\overline{\mathbf{f}}(\overline{\mathbf{x}}, t), \overline{\mathbf{x}}(0)=\overline{\mathbf{x}}_{0}\right\}$.

For any $t \geqslant 0$, the box-valued function $[\phi]\left(\left[\underline{\mathbf{x}}_{0}, \overline{\mathbf{x}}_{0}\right], t\right)=\left[\phi\left(\underline{\mathbf{x}}_{0}, t\right), \bar{\phi}\left(\overline{\mathbf{x}}_{0}, t\right)\right]$ is thus an inclusion function for the solution of (1). This function is difficult to evaluate, as usually no explicit expressions are available for $\underline{\phi}\left(\underline{\mathbf{x}}_{0}, t\right)$ and $\bar{\phi}\left(\overline{\mathbf{x}}_{0}, t\right)$. However, using the guaranteed numerical techniques for solving IVPs such as presented in Section 2.1, it is possible to compute tight enclosures of these quantities

$$
\left[\underline{\phi}\left(\underline{\mathbf{x}}_{0}, t\right)\right]=\left[\underline{\underline{\phi}}\left(\underline{\mathbf{x}}_{0}, t\right), \bar{\phi}\left(\underline{\mathbf{x}}_{0}, t\right)\right]
$$


and

$$
\left[\bar{\phi}\left(\overline{\mathbf{x}}_{0}, t\right)\right]=\left[\overline{\bar{\phi}\left(\overline{\mathbf{x}}_{0}, t\right)}, \overline{\bar{\phi}\left(\overline{\mathbf{x}}_{0}, t\right)}\right] .
$$

The main difference with the situation in Section 2.1 is that now the systems to be integrated only imply intervals of infinitesimal width. The function

$$
[[\phi]]([\mathbf{x}], t)=[\underline{\underline{\phi}}(\underline{\mathbf{x}}, t), \overline{\bar{\phi}(\overline{\mathbf{x}}, t)}]
$$

is such that $[\phi]\left(\left[\underline{\mathbf{x}}_{0}, \overline{\mathbf{x}}_{0}\right], t\right) \subset[[\phi]]\left(\left[\underline{\mathbf{x}}_{0}, \overline{\mathbf{x}}_{0}\right], t\right)$ and therefore an inclusion function for the solution of (1), which can be numerically evaluated for any $t \geqslant 0$.

\subsection{Cooperative models and set observers}

Let $\Phi\left(\left[\underline{\mathbf{x}}_{0}, \overline{\mathbf{x}}_{0}\right], t\right)$ be the set of all $\mathbf{x}(t)$ that can be traced back to an initial condition in $\left[\underline{\mathbf{x}}_{0}, \overline{\mathbf{x}}_{0}\right]$. Then if the conditions of Theorem 1 are verified

$$
\mathbf{x}(t) \in \Phi\left(\left[\underline{\mathbf{x}}_{0}, \overline{\mathbf{x}}_{0}\right], t\right) \subset[[\phi]]\left(\left[\underline{\mathbf{x}}_{0}, \overline{\mathbf{x}}_{0}\right], t\right) \text { for any } t \geqslant 0 .
$$

Interval observers using $[[\phi]]\left(\left[\underline{\mathbf{x}}_{0}, \overline{\mathbf{x}}_{0}\right], t\right)$ are only able to provide a box containing $\Phi\left(\left[\underline{\mathbf{x}}_{0}, \overline{\mathbf{x}}_{0}\right], t\right)$. However, $\Phi\left(\left[\underline{\mathbf{x}}_{0}, \overline{\mathbf{x}}_{0}\right], t\right)$ is usually not a box, see Figure 2 . Here, we propose to improve the accuracy of the approximation of $\Phi\left(\left[\underline{\mathbf{x}}_{0}, \overline{\mathbf{x}}_{0}\right], t\right)$ by enclosing it in a subpaving using the IMAGESP algorithm presented in [15] and [16] and briefly recalled now.

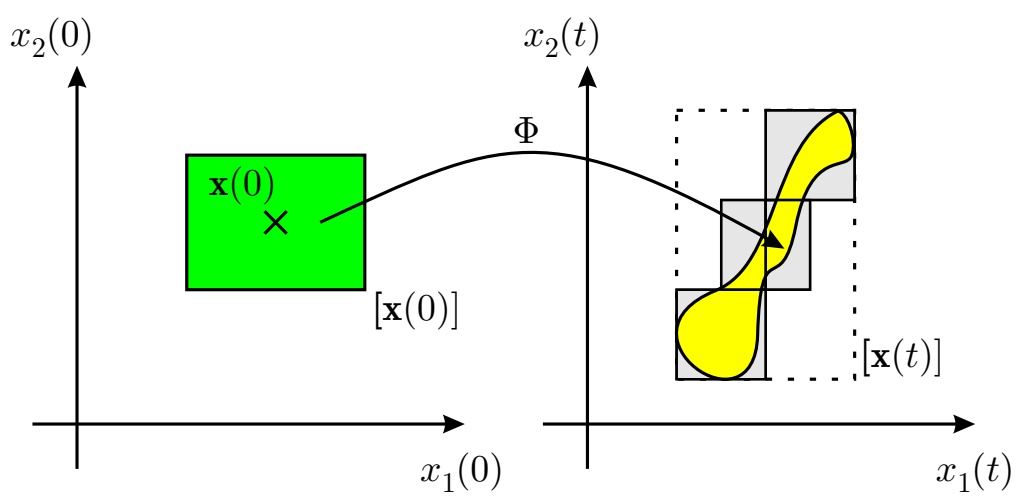

Figure 2: Estimates obtained by an interval observer (box in dashed lines) and an approximate set observer (union of light grey boxes on the right)

IMAGESP consists of three steps. First, $\left[\underline{\mathbf{x}}_{0}, \overline{\mathbf{x}}_{0}\right]$ is minced, i.e., divided into boxes with width less than a given precision parameter $\varepsilon_{\mathrm{I}}$. The images of all these boxes are then evaluated using an inclusion function of $\Phi$ and stored into a list $\mathcal{L}$ of image boxes. Finally, all boxes in $\mathcal{L}$ are merged to obtain a subpaving guaranteed to contain $\Phi\left(\left[\underline{\mathbf{x}}_{0}, \overline{\mathbf{x}}_{0}\right], t\right)$. The time needed to obtain this subpaving and the precision of the description (measured, e.g., using its Hausdorff distance to the approximated set) increase when the precision parameter $\varepsilon_{\mathrm{I}}$ decreases.

The only requirement for IMAGESP is the availability of an inclusion function for $\Phi$, which is obtained using $[[\phi]]$.

\subsection{Implementable algorithm}

Assume that $\mathcal{X}(t)$ has to be evaluated, with $t$ such that $t=t_{N}$, and that $\mathcal{X}\left(t_{0}\right)=\left[\mathbf{x}_{0}\right]$. The following algorithm is an implementable counterpart to Algorithm 1.

Algorithm 2

For $i=0$ to $N$, do \{ 
1. Prediction: evaluate $\widehat{\mathcal{X}}^{+}\left(t_{i+1}\right)$ using IMAGESP;

2. Correction: evaluate $\widehat{\mathcal{X}}\left(t_{i+1}\right)$ using SIVIA with initial search domain $\widehat{\mathcal{X}}^{+}\left(t_{i+1}\right)$; $\}$

Convergence properties have been established in [13] for Sivia and in [16] for ImaGeSp. The convergence of Algorithm 2 does not only depend on $\varepsilon_{\mathrm{S}}$ and $\varepsilon_{\mathrm{I}}$, but also on the quality of enclosure of (1) provided by the pair of cooperative systems.

\section{Example}

This example corresponds to the estimation of the state of an activated-sludge process. It is inspired from [5] and [6], where the model has been introduced. The performance of the interval observer of [5] will be compared to that of the set observer presented here.

Figure 3 illustrates the principle of an activated-sludge process. Waste water enters the aerator. Substrate (waste) is transformed in the aerator by a bacterial population (the biomass). In the settler, sedimentation takes place, where cleared water is separed from the biomass. A part of the biomass is then recycled by reinjection in the aerator. For more details, see [23].

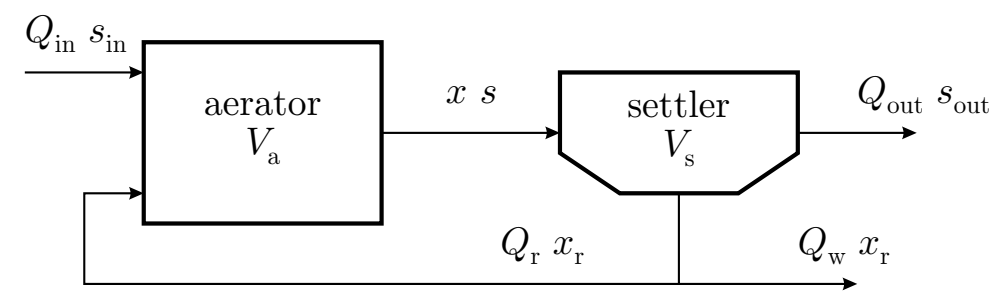

Figure 3: Activated sludge process

The state variables $x, s$ and $x_{\mathrm{r}}$ respectively represent the concentrations of biomass, substrate and recycled biomass. The input and output substrate concentrations are denoted by $s_{\text {in }}$ and $s_{\text {out }} . V_{\text {a }}$ and $V_{\mathrm{s}}$ represent the volumes of the aerator and settler. $Q_{\text {in }}$ and $Q_{\text {out }}$ are the flow rates at the input and output of the system. $Q_{\mathrm{r}}$ and $Q_{\mathrm{w}}$ are the recycled and non-recycled flow rates.

Mass balances of substrate and biomass result in the following set of equations [6]

$$
\left\{\begin{array}{l}
x^{\prime}=\mu(.) x-(1+r) D(t) x+r D(t) x_{\mathrm{r}} \\
s^{\prime}=-\frac{\mu(.) x}{Y_{\mathrm{s}}}-(1+r) D(t) s+D(t) s_{\mathrm{in}} \\
x_{\mathrm{r}}^{\prime}=v(1+r) D(t) x-v(w+r) D(t) x_{\mathrm{r}}
\end{array}\right.
$$

where

$$
\begin{gathered}
D(t)=Q_{\text {in }} / V_{\mathrm{a}}, r=Q_{\mathrm{r}} / Q_{\text {in }}, w=Q_{\mathrm{w}} / Q_{\mathrm{in}}, v=V_{\mathrm{a}} / V_{\mathrm{s}}, \\
x(0)=x_{0}, s(0)=s_{0}, s_{\mathrm{r}}(0)=s_{\mathrm{r} 0} .
\end{gathered}
$$

In (13), $Y_{\mathrm{s}}$ is the yield coefficient of biomass growth on substrate. The specific growth rate $\mu($. depends on many parameters and is usually not well known. The concentrations of biomass $x$ and recycled biomass $x_{\mathrm{r}}$ must be estimated under the following assumptions : the substrate concentration $s$ is known, $Y_{\mathrm{s}}, r, w, v$ are constant and known, $\mu($.$) is unknown, s_{\text {in }}$ is unknown, but its lower and upper bounds $\left(\underline{s}_{\text {in }}\right.$ and $\left.\bar{s}_{\text {in }}\right)$ are known and $D(t)$ satisfies $\underline{d} \leqslant D(t) \leqslant \bar{d}$ for any $t \geqslant 0$, where $\underline{d}$ and $\bar{d}$ are known.

To obtain an interval observer, the unknown growth rate $\mu($.$) is first eliminated in (13) by$ the linear change of variables $\mathbf{x}=\mathbf{z}+\mathbf{K} s$, with

$$
\mathbf{x}=\left(\begin{array}{c}
x_{1} \\
x_{2}
\end{array}\right), \mathbf{z}=\left(\begin{array}{c}
x \\
x_{\mathrm{r}}
\end{array}\right), \mathbf{K}=\left(\begin{array}{c}
Y_{\mathrm{s}} \\
0
\end{array}\right) .
$$


The following reduced linear system is then obtained

$$
\left\{\begin{array}{l}
x_{1}^{\prime}(t)=D(t)\left(-(1+r) x_{1}(t)+r x_{2}(t)+s_{\text {in }}(t)\right), \\
x_{2}^{\prime}(t)=D(t)\left(v(1+r) x_{1}(t)-v(w+r) x_{2}(t)-v(1+r) s(t)\right) .
\end{array}\right.
$$

As lower and upper bounds are known for $s_{\text {in }}(t)$ and $D(t)$ it is possible, according to Theorem 1 , to enclose (14) between two cooperative systems, and thus to build the interval observer

$$
\left\{\begin{array}{l}
\underline{\mathbf{x}}^{\prime}=\underline{\underline{d}}(\mathbf{A} \underline{\mathbf{x}}+\underline{\underline{\mathbf{b}}}(s)) \\
\overline{\mathbf{x}}^{\prime}=\bar{d}(\mathbf{A} \overline{\mathbf{x}}+\overline{\overline{\mathbf{b}}}(s)) \\
\underline{\mathbf{z}}=\mathbf{x}-\mathbf{K} s, \underline{\mathbf{x}}_{0}=\underline{\mathbf{z}}_{0}+\mathbf{K} s_{0} \\
\overline{\mathbf{z}}=\overline{\mathbf{x}}-\mathbf{K} s, \overline{\mathbf{x}}_{0}=\overline{\mathbf{z}}_{0}+\mathbf{K} s_{0}
\end{array}\right.
$$

with

and

$$
\mathbf{A}=\left(\begin{array}{cc}
-(1+r) & r \\
v(1+r) & -v(w+r)
\end{array}\right)
$$

$$
\underline{\mathbf{b}}(s)=\left(\begin{array}{l}
\underline{s}_{\text {in }}(t) \\
-v(1+r) s
\end{array}\right) Y_{\mathrm{s}}, \overline{\mathbf{b}}(s)=\left(\begin{array}{l}
\bar{s}_{\text {in }}(t) \\
-v(1+r) s
\end{array}\right) Y_{\mathrm{s}} .
$$

The performance of this interval observer will be compared to that obtained with the state estimator of Section 2.3. In both cases, $s(t)$ is assumed to be known. No other quantity is measured, so no correction step has to be implemented in this special case. The data have been generated by simulation with numerical values of the parameters taken from [5] :

$$
\begin{gathered}
\mu(.)=\mu_{\max } \frac{s}{K_{\mathrm{s}}+s}, \text { with } \mu_{\max }=0.15 \mathrm{~h}^{-1} \text { and } K_{\mathrm{s}}=100 \mathrm{mg} / \mathrm{l}, \\
Y_{\mathrm{s}}=0.65, v=1, r=0.6, w=0.2, \\
D(t)=0.1, \\
s_{\text {in }}(t)=50 \sin (2 \pi t / 100)+250, \\
\bar{s}_{\text {in }}(t)-\underline{s}_{\text {in }}(t)=60 .
\end{gathered}
$$

The actual initial state for the generation of the data is taken as $s(0)=50 \mathrm{mg} / \mathrm{l}, x(0)=200$ $\mathrm{mg} / \mathrm{l}, x_{\mathrm{r}}(0)=400 \mathrm{mg} / \mathrm{l}$, but the only available knowledge about $x(0)$ and $x_{\mathrm{r}}(0)$ is that

$$
[x(0)]=[0,1000] \mathrm{mg} / \mathrm{l},\left[x_{\mathrm{r}}(0)\right]=[200,1400] \mathrm{mg} / \mathrm{l} .
$$

Subpavings and the prediction step of the state estimator have been implemented according to [11, Chap. 11], and VNODE [21] has been used for guaranteed numerical integration. The parameters $\varepsilon_{\mathrm{S}}$ and $\varepsilon_{\mathrm{I}}$ are tuned at each iteration to keep the number of boxes in each subpaving below $N_{\mathrm{b}}$. This strategy allows the computing time at each step to remain approximatively constant. For various $N_{\mathrm{b}}$, the total computing times of a simulation from $t=0 \mathrm{~h}$ to $t=200 \mathrm{~h}$, on an Athlon 1800 are given in Table 1. These computing times are compatible with the time

\begin{tabular}{c|c|c|c}
$N_{\mathrm{b}}$ & 100 & 500 & 2000 \\
\hline Time $(\mathrm{mn})$ & 20 & 100 & 400
\end{tabular}

Table 1: State estimator computing times

constants of the process. The subpavings obtained for different time instants and $N_{\mathrm{b}}=2000$ are described on Figures 4 and 5 . The box estimates evaluated by the interval observer are the smallest boxes containing these sets. Notice that when the set observer is used in place of the interval observer, any measurement on $x$ (resp. $x_{\mathrm{r}}$ ) would improve the knowledge on $x_{\mathrm{r}}$ (resp. $x$ ). This would not be the case if the interval observer were used.

The much superior accuracy of the set estimator over the interval observer is evidenced in Figure 6, where the evolution of the volumes of the estimates are compared. 

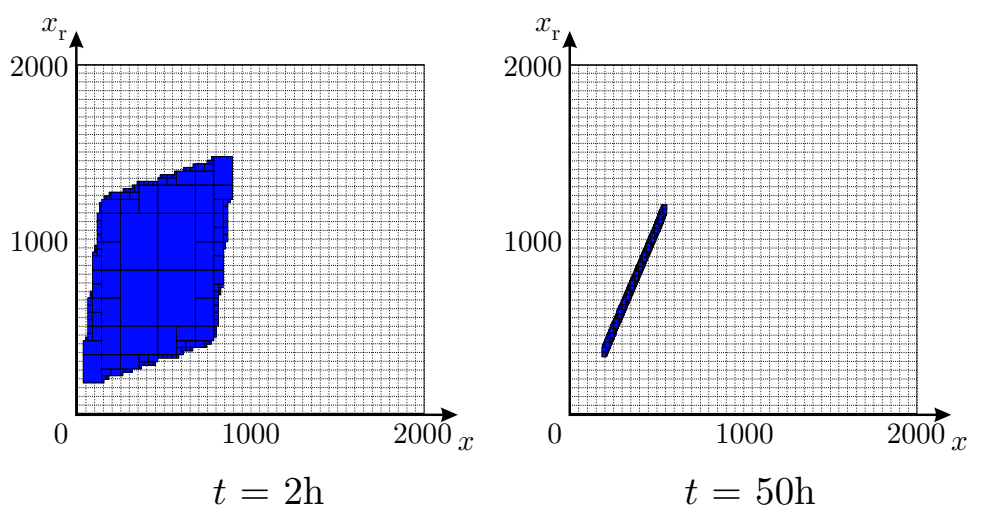

Figure 4: Set estimate for $\mathbf{x}(t)$ at $t=2 \mathrm{~h}$ and $t=50 \mathrm{~h}$
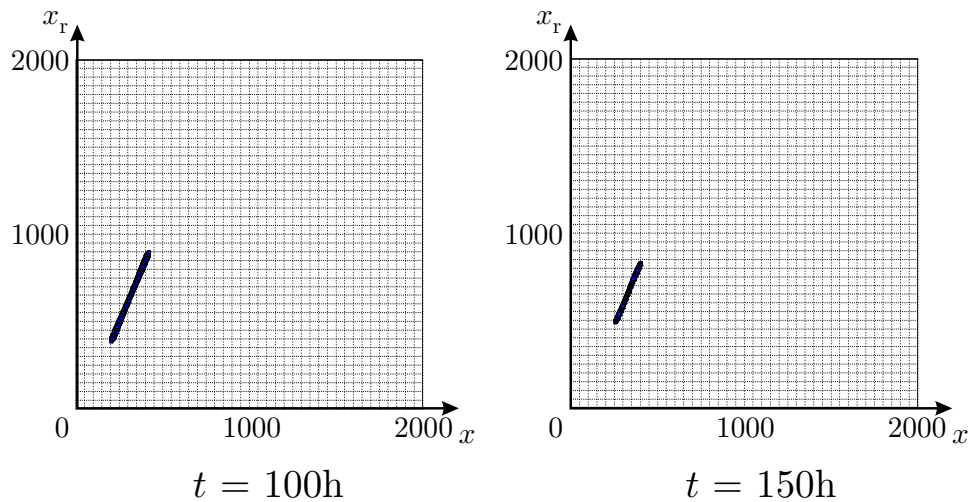

Figure 5: Set estimate for $\mathbf{x}(t)$ at $t=100 \mathrm{~h}$ and $t=150 \mathrm{~h}$

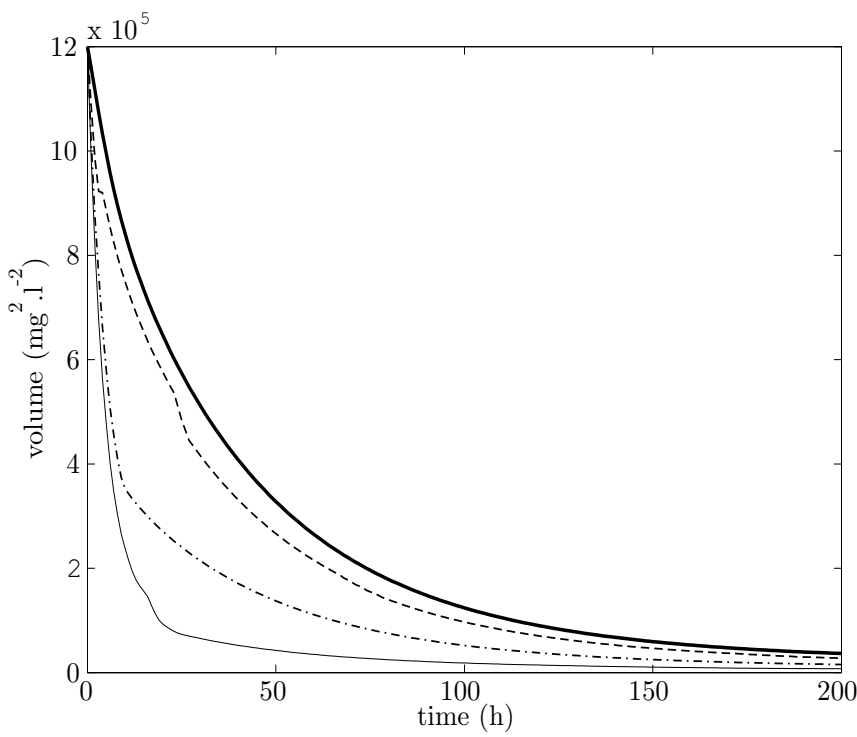

Figure 6: Volume of the estimates provided by the interval observer (bold solid line) and the state estimator for various values of $N_{\mathrm{b}}\left(N_{\mathrm{b}}=100\right.$ : dashed line; $N_{\mathrm{b}}=500$ : dashed-dotted line; $N_{\mathrm{b}}=2000$ : thin solid line) 


\section{Conclusions}

A new recursive non-linear state estimator for continuous-time systems that may be enclosed between cooperative systems has been described. As no linearization of the state equation is performed, the algorithm computes a set guaranteed to contain the actual state, provided that the assumptions on the noise corrupting the state and observation equations are valid. The performances of the set estimator have been compared to those of an interval observer, and it has been shown that it allowed a much more accurate estimation. The main limitation of this estimator is that its complexity grows exponentially with the dimension of the state, which restricts its application to low-dimensionnal problems, such as those encountered, e.g., in biology or pharmacokinetics. Joint state and parameter estimation may be considered with the same tools, provided that the obtained extended-state equation can still be enclosed by cooperative systems.

\section{References}

[1] V. Alcaraz-González, A. Genovesi, J. Harmand, A. González, A. Rapaport, and J. Steyer. Robust exponential nonlinear interval observer for a class of lumped models useful in chemical and biochemical engineering. Application to a wastewater treatment process. In Proc. MISC'99 Workshop on Applications of Interval Analysis to Systems and Control, pages 225-235, Girona, February 24-26, 1999.

[2] F. L. Chernousko. State Estimation for Dynamic Systems. CRC Press, Boca Raton, FL, 1994.

[3] A. Gelb. Applied Optimal Estimation. MIT Press, Cambridge, MA, 1974.

[4] J. L. Gouzé, A. Rapaport, and Z. M. Hadj-Sadok. Interval observers for uncertain biological systems. Journal of Ecological Modelling, (133):45-56, 2000.

[5] Z. M. Hadj-Sadok. Modélisation et estimation dans les bioréacteurs ; prise en compte des incertitudes : application au traitement de l'eau. PhD thesis, Université de Nice-Sophia Antipolis, 1999.

[6] Z. M. Hadj-Sadok, J. L. Gouzé, and A. Rapaport. State observers for uncertain models of activated sludge processes. In CD-Rom of the IFAC-EurAgEng International Workshop on Decision and Control in Waste Bio-Processing, Narbonne, February 25-27, 1998.

[7] J. Hoefkens, M. Berz, and K. Makino. Efficient high-order methods for ODEs and DAEs. In G. Corliss, C. Faure, and A. Griewank, editors, Automatic Differentiation : From Simulation to Optimization, pages 341-351, New-York, NY, 2001. Springer-Verlag.

[8] J. Hoefkens, M. Berz, and K. Makino. Verified high-order integration of DAEs and ODEs. In W. Kraemer and J. W. von Gudenberg, editors, Scientific Computing, Validated Numerics, Interval Methods, pages 281-292, Boston, 2001. Kluwer.

[9] L. Jaulin. Nonlinear bounded-error state estimation of continuous-time systems. Automatica, 38:1079-1082, 2002.

[10] L. Jaulin, M. Kieffer, I. Braems, and E. Walter. Guaranteed nonlinear estimation using constraint propagation on sets. International Journal of Control, 74(18):1772-1782, 2001.

[11] L. Jaulin, M. Kieffer, O. Didrit, and E. Walter. Applied Interval Analysis. Springer-Verlag, London, 2001.

[12] L. Jaulin and E. Walter. Guaranteed nonlinear parameter estimation from bounded-error data via interval analysis. Mathematics and Computers in Simulation, 35(2):123-137, 1993.

[13] L. Jaulin and E. Walter. Set inversion via interval analysis for nonlinear bounded-error estimation. Automatica, 29(4):1053-1064, 1993.

[14] M. Kieffer. Estimation ensembliste par analyse par intervalles, application à la localisation d'un véhicule. PhD thesis, Université Paris-Sud, Orsay, France, 1999. 
[15] M. Kieffer, L. Jaulin, I. Braems, and E. Walter. Guaranteed set computation with subpavings. In W. Kraemer and J. W. von Gudenberg, editors, Scientific Computing, Validated Numerics, Interval Methods, pages 167-178, Boston, 2001.

[16] M. Kieffer, L. Jaulin, and E. Walter. Guaranteed recursive nonlinear state bounding using interval analysis. International Journal of Adaptative Control and Signal Processing, 6(3):193-218, 2002.

[17] A. Kurzhanski and I. Valyi. Ellipsoidal Calculus for Estimation and Control. Birkhäuser, Boston, MA, 1997.

[18] R. Lohner. Enclosing the solutions of ordinary initial and boundary value problems. In E. Kaucher, U. Kulisch, and C. Ullrich, editors, Computer Arithmetic: Scientific Computation and Programming Languages, pages 255-286. BG Teubner, Stuttgart, 1987.

[19] R. Lohner. Computation of guaranteed enclosures for the solutions of ordinary initial and boundary value-problem. In J. R. Cash and I. Gladwell, editors, Computational Ordinary Differential Equations, pages 425-435, Oxford, 1992. Clarendon Press.

[20] M. Milanese, J. Norton, H. Piet-Lahanier, and E. Walter, editors. Bounding Approaches to System Identification. Plenum Press, New York, NY, 1996.

[21] N. S. Nedialkov and K. R. Jackson. Methods for initial value problems for ordinary differential equations. In U. Kulisch, R. Lohner, and A. Facius, editors, Perspectives on Enclosure Methods, pages 219-264, Vienna, 2001. Springer-Verlag.

[22] H. L. Smith. Monotone Dynamical Systems: An Introduction to the Theory of Competitive and Cooperative Systems, volume 41 of Mathematical Surveys and Monographs. American Mathematical Society, Providence, RI, 1995.

[23] G. Stephanopoulos. Chemical Process Control. An Introduction to Theory and Practice. Prentice-Hall, Englewood Cliffs, N. J., 1984. 